\title{
Evidências Empíricas da Lei de Kaldor-Verdoorn para a Indústria de Transformação do Brasil (1985-1997)*
}

\author{
Emerson Luís Lemos Marinho ** \\ Cláudio André Gondim Nogueira *** \\ Antônio Lisboa Teles da Rosa
}

Sumário: 1. Introdução; 2. A lei de Kaldor-Verdoorn: aspectos conceituais e teóricos; 3 . A indústria brasileira: estrutura e movimentos recentes; 4. O modelo econométrico: formalização e análise dos resultados; 5. Conclusão

Palavras-chave: estrutura industrial; produção; produtividade.

Códigos JEL: CS1 e 049.

Este artigo discute a Lei de Kaldor-Verdoorn que propõe que à medida que a produção de um país aumenta há uma forte tendência ao longo do tempo de crescimento da produtividade. Assim, efetuase um teste empírico para a indústria de transformação do Brasil, no período de 1985 a 1997, através de um modelo de correção de erros e de um teste de causalidade de Granger, cujos resultados obtidos indicam um razoável grau de dinamismo, principalmente após a abertura econômica, quando uma combinação de efeitos de curto e longo prazo fez com que a produtividade crescesse num ritmo bem mais acelerado.

In this paper, we have tried to discuss theoretically Kaldor-Verdoorn's Law, showing its relevance to the determination of how dynamic an industry of country on region can be. Furthermore, we have made an empirical test of such theoretical proposition for the Brazilian industry during the period 1985 to 1997, using an error correction model (MCE), whose results indicate that the Brazilian industry has been reasonably dynamic, specially after the opening process of its economy had begun, in 1990, when a combination of

\footnotetext{
*Artigo recebido em set. 1999 e aprovado em jul. 2001. Os autores são gratos aos pareceristas anônimos pelos seus comentários e sugestões, responsabilizando-se inteiramente pelos erros remanescentes

${ }^{* *}$ Doutor em Economia pela EPGE/FGV/RJ e professor do Curso de Pós-Graduação em Economia do Caen/UFC.

*** Doutorando em Economia pelo Curso de Pós-Graduação em Economia do Caen/UFC e professor da Universidade de Fortaleza.

${ }^{* * * *}$ Doutor em Economia pelo Pimes/UFPE e professor do Curso de Pós-Graduação em Economia do Caen/UFC.
} 
short and long run effects made productivity grow more intensively than production.

\section{Introdução}

O artigo de Verdoorn, publicado em 1951 na revista Econometrica, e o de Kaldor, publicado em The Economic Journal em 1975, estabeleceram as bases teóricas para uma relação empírica importante verificada entre o crescimento da produção e o da produtividade do trabalho. Segundo esses autores, à medida que a produção aumenta há uma forte tendência, ao longo do tempo, de que a produtividade do trabalho venha a acompanhar este movimento. Indubitavelmente, uma série de fatores pode ser considerada importantes para explicar esta tendência que, devido à sua relevância, assumiu na literatura o status de lei. Assim, o objetivo deste artigo será justamente o de aprofundar a discussão a respeito da chamada lei de Kaldor-Verdoorn e apresentar evidências empíricas de sua ocorrência para a indústria de transformação do Brasil no período 1985-1997. Para isto, será feita uma análise do comportamento da produção e da produtividade da indústria de transformação do Brasil, por meio de testes de raízes unitárias e de co-integração e de um modelo de correção de erros.

Além desta introdução, o artigo é composto de mais quatro seções. Na seção 2, faz-se uma discussão teórica sucinta sobre a lei de Kaldor-Verdoorn. Na seção 3, apresenta-se uma breve análise sobre o estágio atual de desenvolvimento da indústria brasileira. $\mathrm{Na} \mathrm{4}$, realiza-se a análise empírica com a apresentação do modelo econométrico adotado e os seus principais resultados. Na última, são apresentadas as conclusões finais. O anexo contém as tabelas dos testes estatísticos

\section{A Lei de Kaldor-Verdoorn: Aspectos Conceituais e Teóricos}

A discussão acerca da Lei de Kaldor-Verdoorn inicia-se justamente através de considerações sobre a importância do setor industrial para o crescimento econômico de um país ou região. Isto se dá porque, entre as manifestações mais importantes do processo de crescimento, merecem destaque as transformações que ocorrem ao longo do tempo na estrutura produtiva. Tais transformações provocam alterações bastante significativas na composição da demanda, aumentando a participação dos produtos manufaturados. Ademais, são maiores os estímulos para a adoção de novos processos produtivos e para a criação de novos produtos, gerando, assim, relevantes avanços no lado da oferta. Tal processo como um todo beneficia o 
crescimento da produtividade industrial de uma forma bem mais intensa que em outros setores.

Obviamente, o ganho de importância do setor industrial é apenas uma parte do processo de crescimento econômico. Mas, uma parte muito importante, deve-se salientar. Logicamente, a participação de outros setores neste processo não pode ser de forma alguma desprezada, como é o caso do setor de serviços. Entretanto, o argumento básico aqui desenvolvido é que, especialmente para os países subdesenvolvidos, um maior dinamismo da indústria é fundamental para estimular o crescimento econômico, pois este setor guarda uma inter-relação dinâmica com outros. O setor primário é um dos principais fornecedores de matérias-primas para a indústria. Já o desenvolvimento do setor de serviços (serviços financeiros, manutenção industrial, transportes, comércio etc.) apresenta uma elevada correlação com a evolução do setor industrial: os casos de diversos países desenvolvidos, em que o setor de serviços está em um estágio bastante avançado, tais como EUA, Reino Unido e Japão, parecem indicar. Assim, faz-se uma suposição básica de que o aumento da produção industrial ao longo do tempo deve ser considerado como um dos fatores relevante para se determinar o dinamismo de uma economia.

A partir desta idéia básica, pode-se constatar também que a produtividade do trabalho na indústria está intrinsecamente relacionada com o crescimento da produção. Quando a produção cresce, ocorrem, ao longo do tempo, relevantes transformações na estrutura produtiva e na composição da demanda. Tais transformações vêm a beneficiar a indústria, pois induzem a utilização de novos processos produtivos, bem como o surgimento de novos produtos, fatores essenciais para o crescimento da produtividade.

Mais especificamente no nível micro, quando a produção e a produtividade estão crescendo acima da média em um determinado setor, este movimento tende a ser associado com a queda dos seus custos relativos e, conseqüentemente, com a queda dos seus preços relativos, aumentando, assim, a demanda pelo produto em questão. Já no nível macro, quando todo o setor industrial de um país ou região é considerado, uma maior produtividade freqüentemente faz com que as exportações tornem-se mais competitivas, porque ficam mais baratas e/ou porque os produtos passam a apresentar um melhor nível de qualidade. Desta forma, mais exportações levam ao crescimento do produto industrial, na medida em que se eleva a demanda pelos produtos exportáveis, e isso tem um efeito indireto, pois o crescimento das exportações gera recursos adicionais para financiar as importações necessárias ao crescimento mais acelerado do produto. Adicionalmente, um nível mais elevado 
de produtividade pode, ainda, significar maiores lucros para as firmas, o que também pode afetar positivamente a demanda, na medida em que estas firmas podem investir mais.

Como Rowthorn (1975a) menciona, ${ }^{1}$ "uma maior produtividade na indústria pode estimular a demanda doméstica por produtos industriais, por torná-los relativamente mais baratos ou porque novos produtos são introduzidos no mercado. A maquinaria torna-se mais barata em relação ao trabalho, encorajando a adoção de técnicas mais mecanizadas. Os produtos industrializados tornam-se, então, mais baratos em relação a um grande número de serviços cuja produtividade cresceu lentamente, encorajando a substituição desses serviços por produtos industrializados".

Além disto, há o surgimento de novas unidades empresariais e/ou a ampliação das existentes, o que possibilita o emprego de equipamentos mais modernos, possivelmente mais adequados às unidades produtivas de maior tamanho. Em decorrência disso, espera-se que haja uma relação direta entre o crescimento da produção e da produtividade. E isto é justamente o que propõe a chamada lei de Kaldor-Verdoorn (Verdoorn, 1951, 1956, 1980, Kaldor, 1975, Rowthorn, 1975a,b, 1979).

Uma maneira de se mostrar esta inter-relação formalmente é apresentada por Verdoorn (1956). O ponto de partida é uma função de produção agregada do tipo

$$
y=y\left(x_{1}, \ldots, x_{n}\right)
$$

onde $y$ representa o produto e $x_{1}, \ldots, x_{n}$ representam fatores de produção. São feitas, então, algumas suposições adicionais, tais como:

- todos os fatores apresentam derivadas parciais contínuas, $\partial y / \partial x_{i}, i=1, \ldots, n$;

- o mercado atua em competição perfeita de forma que os preços dos fatores sejam iguais ao seu produto marginal, gerando, assim, $n$ funções demanda do tipo $p_{i}=\partial y / \partial x_{i}$, onde $p_{1}, \ldots, p_{n}$ representam os preços dos fatores;

- do lado da oferta existem $n$ equações do tipo $x_{i}=x_{i}\left(p_{i}, z_{i r}\right)$, onde $z_{i r}$ representa um grupo de variávies exógenas com $r=1, \ldots, m$.

Desta forma, fazendo-se adicionalmente a hipótese essencial de complementariedade entre os fatores de produção, pode-se, então, determinar o equilíbrio, de forma que apenas uma combinação de fatores a cada nível de produto seja admissível, como em:

\footnotetext{
${ }^{1}$ Tradução livre feita pelos autores.
} 


$$
x_{i}=x_{i}(y)
$$

onde $x_{i}(y)$ representa qualquer função que relacione $x_{i}$ unicamente a $y .^{2}$

Supõe-se, por exemplo, que $x_{i}$ e $y$ apresentem uma relação de longo prazo da forma

$$
x_{i}=\alpha y^{\beta}
$$

Sem dúvida, esta é uma boa forma de relacionar as variações na produtividade dos fatores quando o produto varia. Segundo Hirsch (apud Verdoorn (1956)), esta forma aplica-se particularmente bem à demanda por trabalho. E, de acordo com pesquisas empíricas realizadas por este autor, a produtividade do trabalho está unicamente relacionada ao volume acumulado de produto, como em:

$$
y / a=\alpha\left(\int_{0}^{t} y d t\right)^{\beta}
$$

onde $a$ representa o fator trabalho.

Como o interesse básico de Verdoorn (1956) é determinar a trajetória de longo prazo das variáveis em questão, então, se for considerado um período razoavelmente longo, $y$ poderá ser aproximado por

$$
y_{t}=\gamma e^{\zeta t}
$$

Substituindo-se este valor no lado direito de (4), encontra-se

$$
y / a=\alpha\left(\frac{y-\gamma}{\zeta}\right)^{\beta}
$$

E, à medida que $t$ aumenta, $y / a$ aproximará a expressão

$$
y / a=\alpha \zeta^{-\beta} y^{\beta}
$$

Assim, encontra-se uma relação estável de longo prazo entre produtividade e o produto de uma economia. E, de acordo com Verdoorn (1956), o mecanismo básico que explica esta relação é: o nível de produto determina a divisão do trabalho na

\footnotetext{
${ }^{2}$ Para mais detalhes acerca das condições de equilíbrio, ver Verdoorn (1956).
} 
economia e a divisão do trabalho gera tanto economias internas (especialização etc.) quanto economias externas (desenvolvimento do estoque de capital humano e da tecnologia).

A partir desta constatação de Verdoorn, esta inter-relação também pode ser expressa como em:

$$
p=c+d q
$$

onde $p$ e $q$ representam as taxas de crescimento da produtividade e do produto, respectivamente, enquanto $c$ e $d$ são constantes com $d>0$. Se $p$ e $q$ são dados em forma logarítmica, encontra-se a expressão definida por Verdoorn em seu artigo seminal. E o coeficiente $d$ seria uma medida da chamada elasticidade produtividadeprodução $(\eta)$, que representa a chamada Lei de Verdoorn, pois

$$
\eta=\frac{d(y / a)}{d y} \frac{y}{(y / a)}=\frac{d(y / a) /(y / a)}{d y / y}=\frac{d p}{d q}=d
$$

Uma maneira de se mostrar formalmente a inter-relação entre o crescimento da produção e o da produtividade em termos desta elasticidade foi desenvolvida por Verdoorn (1980). Conforme este autor, deve-se considerar inicialmente o seguinte sistema de equações:

$$
\begin{gathered}
y_{t}=a_{t}^{\lambda} b_{t}^{\mu} e^{v t} \\
\dot{b}_{t}=\gamma y_{t} \\
a_{t}=a_{0} e^{\pi t}
\end{gathered}
$$

onde $y_{t}$ representa o produto, $a_{t}$ o fator trabalho e $b_{t}$ o fator capital. Verdoorn assume que a função de produção dada pela expressão (10) permite a existência tanto de economias de escala quanto de progresso tecnológico, de forma que $\xi=\lambda+\mu>1$.

A trajetória temporal de $\dot{y}_{t} / y_{t}$, então, toma a forma

$$
\frac{\dot{y}_{t}}{y_{t}}=\frac{(\pi \lambda+v)}{1-\mu} A_{t}
$$


onde $A_{t}$ é dado por

$$
A_{t}=1+\mu \frac{\gamma(1-\mu)-\kappa_{0}(\pi \lambda+v)}{\gamma(1-\mu)\left[e^{(\pi \lambda+v) t}-1\right]+\kappa_{0}(\pi \lambda+v)}
$$

onde $\kappa_{0}$ representa a relação capital-produto em $t=0$.

Como pode ser verificado, $A_{t} \rightarrow 1$ se $t \rightarrow \infty$, fornecendo, assim, os valores de steady state do sistema:

$$
\begin{gathered}
\lim _{t \rightarrow \infty} \frac{\dot{y}}{y}=\lim _{t \rightarrow \infty} \frac{\dot{b}}{b}=\frac{(\pi \lambda+v)}{1-\mu} \\
\lim _{t \rightarrow \infty} \kappa=\frac{\gamma(1-\mu)}{\pi \lambda+v}
\end{gathered}
$$

enquanto que a taxa de crescimento da produtividade e a elasticidade produtividade-produção são dadas por

$$
\begin{gathered}
\lim _{t \rightarrow \infty} d \ln (y / a)=\frac{\pi(\xi-1)+v}{1-\mu} \\
\lim _{t \rightarrow \infty} \frac{d \ln (y / a)}{d \ln y}=\lim _{t \rightarrow \infty} \eta=\frac{\pi(\xi-1)+v}{\pi \lambda+v}
\end{gathered}
$$

Uma questão importante que emerge desta discussão é a seguinte: seria o crescimento da produtividade mais ou menos que proporcional ao aumento da produção industrial?

Conforme Kaldor (1975), este crescimento seria mais que proporcional, pois existiriam economias de escala, em sentido macroeconômico, que garantiriam tal comportamento, principalmente nos países e nas regiões mais desenvolvidas. Isto ocorre porque tais regiões geralmente possuem mercados internos mais dinâmicos, bem como uma maior capacidade exportadora, que permitem que as empresas cresçam ao longo do tempo, incorporando ganhos crescentes de produtividade advindos do desenvolvimento das atividades produtivas, dos conhecimentos dos trabalhadores, das facilidades de difusão de novos conhecimentos, da existência de economias de aglomeração etc.

A representação de Kaldor da lei de Verdoorn (que passou, então, a se chamar lei de Kaldor-Verdoorn) é feita através da associação do crescimento do emprego (e) com o crescimento do produto $(q)$, partindo-se da identidade básica de que $p \equiv q-e$. Assim, ter-se-ia que

$$
e=\omega+\theta q
$$


com $0<\theta<1$. Esta restrição ao parâmetro $\theta$ seria, conforme Kaldor (1975), uma condição suficiente para a presença de economias de escala estáticas e dinâmicas e, também, para que a relação entre e e $q$ seja estatisticamente significativa.

Entre as diversas críticas a esta visão, destacam-se as de Vaciago (apud Lima (1993)) e Rowthorn (1975b), que aceitam que a relação existente entre o crescimento do produto industrial e o da produtividade é positiva, mas também mostram em detalhes que isto ocorre de forma menos intensa que a descrita por Kaldor.

As implicações desta discussão são indubitavelmente muito importantes, pois admitir que a produtividade cresce de forma mais intensa que a produção significa dizer que há uma forte tendência ao aumento das desigualdades entre países ou regiões ricas e pobres, uma vez que os mais ricos incorporariam de forma mais intensa os ganhos de produtividade. Por outro lado, admitir que o crescimento da produtividade nos países mais ricos é paulatinamente mais lento que o crescimento da produção industrial implica dizer que há uma certa convergência da produtividade entre países ou regiões, reduzindo o diferencial de industrialização existente entre eles, haja vista que nos países mais pobres a produtividade tenderia a crescer de forma mais intensa, pois menores seriam as deseconomias de escala existentes. No entanto, deve-se ressaltar que é possível haver deseconomias de escala que poderão fazer com que a produtividade cresça com o produto industrial, mas a taxas decrescentes. Assim, existem dois efeitos contraditórios. Dependendo de qual deles predomine, a industrialização tenderá a ser mais concentrada ou não em determinados países ou regiões.

Desta perspectiva conciliadora e a partir da idéia de que a Lei de KaldorVerdoorn é antes de tudo uma proposição intrinsecamente empírica (apesar do esforço de se mostrar que esta proposição justifica-se também no nível teórico), admite-se que apenas empiricamente é que se pode constatar realmente qual seria a verdadeira relação existente entre o crescimento da produtividade e o do produto.

É justamente desta perspectiva que se propõe buscar evidências empíricas da lei de Kaldor-Verdoorn para a indústria de transformação do Brasil. Antes, porém, deve ser feita uma breve caracterização desta indústria, mencionando aspectos estruturais, bem como seus movimentos recentes, pois assim será mais profícua a interpretação dos resultados encontrados na análise empírica que será feita mais adiante.

\section{A Indústria Brasileira: Estrutura e Movimentos Recentes}

Nos últimos anos, a indústria brasileira de transformação vem passando por uma série de mudanças estruturais bastante significativas. Em meados da década 
de 1980, iniciou-se no país um tímido processo de abertura econômica, que viria a intensificar-se significativamente no início da década de 1990, com o governo Collor.

Começou, então, um amplo processo de reestruturação produtiva na indústria brasileira, para fazer face ao novo ambiente competitivo instaurado, uma vez que o país permaneceu relativamente fechado à concorrência internacional até o final da década de 1980.

Antes do início dos anos 1990, quando se deu a intensificação do processo de abertura econômica e a redução progressiva das alíquotas de importação, aumentando paulatinamente o intercâmbio comercial do Brasil com os vários países do mundo, não existia uma cultura desenvolvida no sentido de privilegiar a inovação tecnológica e os ganhos de produtividade, uma vez que o relativo isolamento da economia brasileira favorecia a um comportamento acomodativo por parte das empresas. Além das dificuldades existentes no que diz respeito ao acesso às importações de bens de capital e insumos, a falta de concorrência desestimulava a busca pela fabricação de produtos qualitativamente melhores e com custos mais baixos.

Isto foi um reflexo das políticas governamentais adotadas até então, que privilegiavam o aprofundamento do processo de substituição de importações, em vez de uma maior integração com as demais economias mundiais, e também devido à forte crise pela qual passou a economia brasileira ao longo da década de 1980 . Este conjunto de fatores provocou um quadro de estagnação da produção industrial e de contração dos investimentos, limitando sensivelmente a capacidade de incorporação de avanços tecnológicos pela indústria e, conseqüentemente, a sua competitividade.

$\mathrm{Na}$ verdade, a estrutura industrial brasileira era (e ainda é) bastante heterogênea, isto é, apesar de possuir empresas ineficientes e tecnologicamente defasadas, encontram-se, também, empresas modernas, tecnologicamente atualizadas e capazes de competir em nível internacional. Mesmo assim, a década de 1980 foi marcada pela estagnação e pelo atraso tecnológico e organizacional em relação aos padrões internacionais em praticamente todos os setores da indústria.

Contudo, no início dos anos 1990, com o aprofundamento do processo de abertura, muitos setores industriais passaram a ver a sua posição ameaçada por concorrentes estrangeiros que fabricam produtos similares aos seus, porém com maior conteúdo tecnológico, com melhor qualidade e, em muitos casos, com preços muitos menores. A estrutura industrial existente até então se viu confrontada por um novo padrão de concorrência, baseado na incorporação intensiva do progresso técnico e na melhoria contínua de produtos e processos produtivos, decorrentes de 
um ambiente no qual apenas as empresas verdadeiramente eficientes sobrevivem.

Nesse contexto, muitas empresas brasileiras ou estrangeiras que operam no país sentiram a necessidade premente de reduzir custos e adotar formas mais eficientes de produção, a fim de conseguir obter um crescimento relevante dos seus níveis de produtividade e, conseqüentemente, de sua competitividade. De acordo com Silva \& Laplane (1994), entre as estratégias utilizadas pelas empresas, destacam-se:

- a redefinição das linhas de produtos;

- a desverticalização da produção, seja através da importação de insumos ou componentes, seja por meio da terceirização;

- a implementação de inovações organizacionais;

- o estabelecimento de acordos com empresas estrangeiras para a atuação no mercado doméstico (complementação de linhas de produtos, prestação de serviços de distribuição e assistência técnica etc.).

Através da análise dos dados do valor real da produção e produtividade industrial brasileira, pode-se verificar que, a partir de abril de 1990, a produção industrial e a produtividade voltam a crescer, o que representa uma significativa quebra estrutural em relação à tendência verificada na década anterior, que era eminentemente declinante. Para se ter um idéia disto, estimações com base nas séries do valor real da produção e da produtividade industrial brasileira indicam que as taxas médias de crescimento no período que vai de janeiro de 1985 até abril de 1990 foram, respectivamente, de $-0,95 \%$ a.m. (-10, $8 \%$ a.a.) e $0-0,23 \%$ a.m. ( $-2,77 \%$ a.a.), enquanto no período de maio de 1990 até dezembro de 1997, as tendências estimadas foram de um crescimento médio de $0,48 \%$ a.m. (5, 97\% a.a.) e $0,23 \%$ a.m. (2, $83 \%$ a.a.). Vale ressaltar que esta evidência é corroborada pelos resultados obtidos do modelo econométrico formulado na próxima seção.

Esta quebra de tendência se pode ser justificada pelo fato de que a indústria brasileira, ao se ajustar ao processo de globalização, tendeu a manifestar uma certa (re)divisão inter-regional do trabalho, na qual cada região tendeu a especializar-se nos setores para os quais demonstram maior vocação, isto é, que apresentam maior capacidade de permanência no mercado, integrando, assim, as vantagens comparativas e competitivas (Rosa, 1998). ${ }^{3}$ Desta forma, cresceu a velocidade de ajuste da indústria de cada região, fazendo com que se acelerasse o processo de convergência

\footnotetext{
${ }^{3}$ A noção de vantagens comparativas está ligada à dotação de fatores de produção em um determinado país ou região. Assim, caso um país ou região disponha de determinado conjunto de fatores em abundância, haverá a tendência à especialização em atividades econômicas que
} 
de produtividade, como mostrado por Bosco et al. (1997). Isto é resultado da maior velocidade com que ocorre a difusão de novas tecnologias, uma vez que os próprios empresários passaram a dispor, mais rapidamente, de maior quantidade de informações a respeito de novos produtos e processos, principalmente a partir do momento em que receberam um choque mais forte de concorrência externa.

Uma questão importante que surge desta análise é se houve ou não uma significativa melhoria qualitativa da indústria de transformação brasileira seguindo esta tendência de recuperação apresentada no período pós-abertura. Entre os vários indicadores de eficiência que podem fornecer evidências nesse sentido, indubitavelmente, a produtividade parece ser um dos mais relevantes e, também, um dos mais utilizados. Assim, torna-se importante verificar empiricamente se o aumento da produção foi acompanhado por aumentos da produtividade, levando em consideração uma quebra estrutural no início dos anos 1990 (abril de 1990), e, assim, observar como a relação produção-produtividade se comportou antes e após dessa quebra estrutural.

Esta relação entre crescimento da produção e da produtividade, conforme foi dito, é expressa teoricamente através da chamada lei de Kaldor-Verdoorn. E, para que seja feito o teste empírico desta proposição teórica, faz-se necessário estudar os comportamentos da produção e da produtividade, inferindo como o comportamento do primeiro indicador afeta o do segundo ao longo do tempo. Isto será realizado com a ajuda de um modelo econométrico, que será apresentado a seguir.

\section{O Modelo Econométrico: Formalização e Análise dos Resulta- dos}

Antes de ser apresentado o modelo econométrico propriamente dito, faz-se necessária uma discussão acerca dos indicadores utilizados. No caso, será utilizado o índice do valor real da produção da indústria de transformação do Brasil como uma proxy para se avaliar o comportamento da produção desta indústria. Quanto à produtividade, pelas razões descritas logo a seguir, utilizou-se seguinte medida de produtividade parcial:

privilegiem a utilização de tais fatores, pois os custos de produção serão relativamente menores do que em países onde estes fatores sejam escassos. Já a noção de vantagens competitivas está ligada a vantagens concorrenciais construídas. Assim, a dotação de fatores deixaria de ser essencial, uma vez que características tecnológicas, organizacionais e logísticas é que passariam a ser preponderantes para a determinação das atividades econômicas nas quais um país ou região tenderia a se especializar (Kenen, 1994, Porter, 1993). 


$$
P M=P / H
$$

onde:

$P M=$ produtividade média do trabalho;

$P=$ valor real da produção;

$H=$ horas trabalhadas (empregadas na produção).

No entanto, alguns comentários devem ser feitos sobre o uso desta medida de produtividade parcial no lugar da PTF (produtividade total dos fatores). Em geral, existem duas abordagens para o cálculo da PTF: a do resíduo de Solow, tradicionalmente usada nos modelos de crescimento, e a realizada através de númerosíndices como os de Malmquist, Tornqvist e Fisher (Coelli et al., 1998). Em relação a esta última abordagem, a menos de retornos constantes de escala, pode-se demonstrar, como em Coelli et al. (1998), que a PTF pode ser decomposta em três componentes, ou seja, $P T F=$ (variação de eficiência técnica)x(efeitos de variação na escala de operação)x(variação tecnológica).

Assim, nos modelos paramétricos, para o cálculo da PTF faz-se necessário estimar uma função de produção. Nos modelos não-paramétricos adota-se a técnica de data envelopment analysis (DEA), que faz uso de programação matemática [Färe et al. (1994)]. Em ambos os casos, tornam-se evidentes as dificuldades e complicações técnicas de seus usos. Nestes termos, por questão de simplicidade, usou-se a produtividade média do trabalho, mas com a ressalva de que ela é apenas uma parcela da variação de eficiência técnica, que, por sua vez, é um componente da PTF. Assim, a análise dos resultados têm de ser feita levando em consideração que o aumento da produtividade total pode também advir dos efeitos de escala e variação tecnológica.

No cálculo da produtividade média do trabalho foi utilizado o número de horas trabalhadas (ou homens-hora) como uma medida do tempo empregado na produção. Esta escolha é justificada através do seguinte exemplo. Suponha dois trabalhadores onde um produz uma unidade de produto em quatro horas e o outro uma mesma unidade produto em 8 horas. A produtividade média do primeiro trabalhador medida em horas trabalhadas seria de 0,25 e, portanto, menor que a do segundo, que é igual a 0,13 . No entanto, se estas produtividades fossem medidas em função do número de trabalhadores, elas seriam iguais. Neste sentido, o uso da produtividade média do trabalho em função das horas trabalhadas reflete mais fielmente a produtividade média do trabalho. Além do mais, a existência de horas extras e greves com produção parcial (menos horas de trabalho) reforça a justificativa para o uso deste indicador. 
Os dados brutos utilizados são os da pesquisa industrial mensal do IBGE (1998). Estes dados são mensais e apresentados sob a forma de um índice (a média de 1985 é igual a 100) no período de janeiro de 1985 a dezembro de 1997. A escolha do período em questão é importante, pois, há a possibilidade de se captar o comportamento do indicador em estudo no estágio anterior ao processo de abertura comercial brasileira, bem como o seu comportamento ao longo de quase sete anos após esse processo haver iniciado.

Deve-se ainda salientar que todos os indicadores cujos comportamentos serão analisados (produção e produtividade média) são apresentadas em logaritmo e foram dessazonalizados de forma que o seu comportamento de longo prazo fosse captado sem sofrer as influências das variações sazonais. O método de ajustamento sazonal utilizado foi o processo multiplicativo das razões de médias móveis. Este método consiste em computar a média móvel anual das séries e, em seguida, calcular as razões entre os valores mensais (que compõem um ano) em relação à média móvel do ano em questão. De posse desses dados, é feita uma média de todas as razões durante todos os anos da amostra para cada mês, separadamente. E essas médias são justamente os efeitos sazonais. Finalmente, são computadas as séries ajustadas, por meio da divisão de seus valores originais pelos fatores sazonais. Vale ainda ser destacar que este método só pode ser adotado quando as séries apresentam estritamente valores positivos, condição esta que é plenamente cumprida no caso das séries em questão.

Em virtude das transformações efetuadas nas séries originais (aplicação dos logaritmos e dessazonalização), estas serão doravante representadas por LPBRSA e LPMBRSA, que representam, respectivamente, os logaritmos do valor bruto da produção e da produtividade média da indústria de transformação do Brasil já dessazonalizadas.

Adicionalmente, antes de se estabelecer qualquer modelagem para as variáveis em questão, é interessante que se analisem as propriedades estocásticas das referidas séries. Neste sentido, a figura 1 descreve graficamente o comportamento das duas variáveis ao longo do tempo.

Observa-se um comportamento decrescente de ambas as séries até os anos 1990 e, a partir desse período, as suas tendências se revertem, passando a crescer ao longo do tempo. Assim, esta evidência parece mostrar que as séries não são estacionárias. Ademais, o estudo de seus correlogramas fornece, também, indícios neste sentido. Para a confirmação estatística da não-estacionariedade das séries realizase, então, o teste de Dickey-Fuller aumentado acrescentando uma constante e uma tendência. As tabelas A.1 e A.2, no anexo, mostram, respectivamente, os resultados deste teste para as variáveis LPBRSA e LPMBRSA. Comparando-se os valores 
obtidos do teste de Dickey-Fuller com os valores críticos de MacKinnon, conclui-se pela aceitação da hipótese nula de que as séries apresentam raízes unitárias, ou em outras palavras, as séries podem ser consideradas não-estacionárias. ${ }^{4}$ Para a obtenção destes resultados, realizou-se este teste com uma a seis defasagens. O que apresentou a menor estatística de acordo com o critério de Akaike foi o teste com quatro defasagens para ambas as variáveis como é mostrado nas tabelas A.1 e A.2.

No entanto, embora se possa considerar que as séries não são estacionárias, suas trajetórias ao longo do tempo, conforme a figura 1, parecem indicar que existe uma relação de equilíbrio de longo prazo entre elas. De fato, as séries apresentam tendências decrescentes a partir do ano de 1985 até abril de 1990, quando, então, passam a crescer. No entanto, observa-se que cada uma delas evolui ao longo do tempo como se estivesse na mesma "onda", apresentando comovimentos semelhantes. Neste sentido, esta evidência parece indicar que as séries produção e produtividade são co-integráveis.

Figura 1

Trajetórias temporais dos logaritmos da produção e da produtividade da indústria de transformação do Brasil

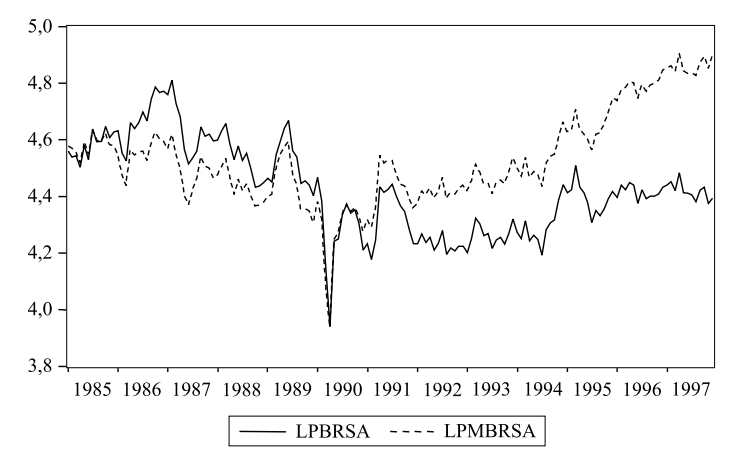

Fonte dos dados brutos: pesquisa industrial mensal do IBGE, 1998.

Para a confirmação estatística desta evidência realiza-se, então, o teste de

\footnotetext{
${ }^{4} \mathrm{Na}$ realidade, os correlogramas das primeiras diferenças de LPBRSA e LPMBRSA parecem indicar que ambas são integradas de ordem 1, os seja, elas são I(1). O teste de Dickey-Fuller aumentado para as primeiras diferenças destas séries apresentaram, respectivamente, os valores $-7,07$ e $-6,98$, os quais, quando comparados, no nível de $5 \%$, com o valor crítico de MacKinnon, de $-3,44$, fornecem indícios que levam à rejeição da hipótese de existência de raízes unitárias nas séries transformadas.
} 
cointegração ${ }^{5}$ proposto por Søren Johansen (Banerjee, 1993, Hill et al., 1999). A tabela 5, no anexo, apresenta os resultados deste teste.

Conclui-se, então, no nível de $5 \%$, que a hipótese nula de não co-integração é rejeitada, existindo, portanto, uma relação de equilíbrio de longo prazo entre produtividade e produção. A análise do vetor co-integrante normalizado revela que esta relação de equilíbrio de longo prazo é dada pela expressão

$$
y_{t}=-87,27+18,69 x_{t}
$$

$y_{t}$ e $x_{t}$ representam, respectivamente, LPMBRSA e LPBRSA.

Assim, verifica-se que existe uma correlação positiva entre produção e produtividade no longo prazo, como preconiza a lei de Kaldor-Verdoorn.

Com base no teste de co-integração e para confirmar a hipótese formulada por Verdoorn e Kaldor, de que esta inter-relação entre produção e produtividade se dá no sentido de que é o crescimento da primeira que influencia o crescimento da segunda, e não o contrário, foi realizado o teste de causalidade de Granger, cujos resultados são apresentados na tabela A.5, no anexo.

De acordo com os resultados do teste, pode-se perceber que a hipótese nula de que a produção não causa a produtividade foi rejeitada, conforme o valor do teste $F$, enquanto que foi aceita a hipótese nula de que a produtividade não causa a produção. Este resultado também refuta, pelo menos para o caso da indústria brasileira, a crítica de Gomulka-Rowthorn à lei de Kaldor-Verdoorn, de que a maneira mais apropriada de se estudar a inter-relação entre o crescimento da produtividade e o crescimento da produção seria através de um sistema de equações simultâneas, uma vez que estes autores percebem que a causalidade entre as variáveis em análise se daria em ambos os sentidos, e não apenas no sentido produção-produtividade (Rowthorn, 1975a,b, 1979, Kaldor, 1975). Assim, os resultados do teste confirmam a causalidade percebida por Verdoorn e por Kaldor e garante a consistência da análise realizada neste artigo.

Segundo o teorema da representação de Granger, um sistema de variáveis co-integráveis pode ser representado de três formas principais: como um vetor

\footnotetext{
${ }^{5}$ Para a escolha do VAR subjacente ao teste Johansen, estimou-se por $M Q O$ as equações $y_{t}=c+\sum_{j=1}^{p} \alpha_{j} y_{t-j}+\sum_{j=1}^{p} \beta_{j} x_{t-j}+\epsilon_{1 t}$ e $x_{t}=c^{\prime}+\sum_{j=1}^{p} \phi_{j} y_{t-j}+\sum_{j=1}^{p} \theta_{j} x_{t-j}+\epsilon_{2 t}$ com $p$ variando de 1 a 6. De acordo com os critérios de Akaike (CA) e Schwarz (CS) para $y_{t-j}$ e $x_{t-j}$, as menores estatísticas $C A=-5,82$ e $C S=-5,73$, para $y_{t-j}$, e $C A=-5.62$ e $C S=-5,56$ para $x_{t-j}$, permitem que o VAR escolhido seja com uma defasagem $(p=1)$. Veja o resultado na tabela A.4 no anexo. Esta mesma estrutura do VAR foi utilizada na realização do teste de causalidade de Granger. As variáveis $y_{t}$ e $x_{t}$ representam, respectivamente, LPMBRSA e LPBRSA.
} 
auto-regressivo (VAR), como um mecanismo de correção dos erros (MCE) e como médias-móveis. O teorema mostra, inclusive, que estas representações são todas isomórficas duas a duas (veja, por exemplo, uma demonstração em Banerjee (1993)).

Nestes termos, supondo que o processo gerador dos dados (PGD) da produtividade, $y_{t}$, seja um modelo de defasagens distribuídas auto-regressivo linear com duas defasagens e uma variável exógena $[D D A(2,2 ; 1)]$, ou seja,

$$
y_{t}=\alpha_{0}+\alpha_{1} y_{t-1}+\alpha_{2} y_{t-2}+\beta_{10} x_{t}+\beta_{11} x_{t-1}+\beta_{12} x_{t-2}+\epsilon_{t}
$$

tem-se que uma representação de (22) na forma do modelo de correção de erros (MCE) é dada por:

$$
\Delta y_{t}=\alpha_{0}+\eta_{1}\left(y_{t-1}-x_{t-1}\right)+\eta_{2}\left(y_{t-2}-x_{t-2}\right)+\beta_{10} \Delta x_{t}+\delta_{11} x_{t-1}+\delta_{12} x_{t-2}+\epsilon_{t}
$$

onde: $\eta_{1}=\alpha_{1}-1, \eta_{2}=\alpha_{2}, \delta_{11}=\alpha_{1}-1+\beta_{10}+\beta_{11}, \delta_{12}=\alpha_{2}+\beta_{12}$ e $\Delta$ o operador de diferenças, tal que $\Delta y_{t}=y_{t}-y_{t-1}$. As inovações $\epsilon_{t^{\prime}} \mathrm{s}$, por hipótese, são independentes e identicamente distribuídas com média 0 e variância $\sigma^{2}$.

Vale salientar que, para que a representação do modelo de defasagens distribuídas auto-regressivo linear sob a forma de um MCE seja apresentada como em (23), deve-se adotar uma hipótese fundamental sobre os termos de correção de erros que, formalmente, são apresentados da seguinte forma: $\left(y_{t-i}-\theta x_{t-i}\right)$. Desde que $\theta \neq 1$, a hipótese adotada é de não homogeneidade. No entanto, conforme Banerjee (1993), mesmo que $\theta$ seja desconhecido, pode-se permitir a inclusão do termo de correção de erros, $\left(y_{t-i}-\theta x_{t-i}\right)$, através da inclusão de defasagens extras de $x_{t}$, sem prejuízo na magnitude dos coeficientes estimados de $\eta_{i}$ em (23). A conclusão é que $\eta_{i}$ reflete diretamente os ajustes dos desequilíbrios mesmo que o verdadeiro termo de correção de erros seja $\left(y_{t-i}-\theta x_{t-i}\right)$ e não $\left(y_{t-i}-x_{t-i}\right)$. Daí, a justificativa para a inclusão dos termos $\delta_{11} x_{t-1}$ e $\delta_{12} x_{t-2}$ em (23).

No nível teórico, as vantagens de se utilizar um MCE são descritas com muita propriedade por Kennedy (1998). Segundo este autor, as principais propriedades destes modelos são:

- sua estrutura permite incorporar informações provenientes da teoria econômica acerca das forças que conduzem ao equilíbrio de longo prazo, uma vez que os modelos econométricos tradicionais são, em sua maioria, essencialmente estáticos; 
- ele admite a adoção muito flexível de defasagens (lags), permitindo que os dados desempenhem um papel fundamental na especificação da estrutura dinâmica dos modelos.

Já no nível prático, a grande vantagem de utilizar um modelo de correção de erros como este para se buscar uma relação empírica entre os impactos do crescimento da produção sobre a produtividade está no fato de que ele fornecerá estimativas da referida elasticidade produtividade-produção, tanto no curto quanto no longo prazo, como será visto mais adiante. Assim, com a separação dos efeitos, a análise dos resultados poderá ser muito mais profícua, melhorando a qualidade de explicação do modelo proposto.

Na equação (23), o termo $\beta_{10}$, que está associado à primeira diferença da variável independente, $x_{t}$, representa a relação de curto prazo entre produtividade e produção. Já os coeficientes $\eta_{1}$ e $\eta_{2}$, associados respectivamente aos termos de correção de erros $\left(y_{t-1}-x_{t-1}\right)$ e $\left(y_{t-2}-x_{t-2}\right)$, medem a relação de longo prazo entre elas.

No entanto, antes de se estimar a equação (23), verifica-se, na análise das trajetórias das variáveis envolvidas na figura 1, que parece haver uma quebra estrutural em abril de 1990. Aliás, deve-se salientar que foi no início dos anos 1990 que o Brasil começou a abrir sua economia, incrementando suas relações comerciais com o resto do mundo. Neste sentido, realiza-se a seguir um teste de hipótese utilizando variáveis dummies ${ }^{6}$ para detectar se estatisticamente ocorre quebra estrutural neste período. Assim, introduzindo estas variáveis na equação (23), obtém-se o seguinte modelo:

$$
\begin{aligned}
\Delta y_{t} & =\alpha_{0}+\alpha_{1} D_{t}+\eta_{1} D_{t}\left(y_{t-1}-x_{t-1}\right) \\
& +\eta_{2} D_{t}\left(y_{t-2}-x_{t-2}\right)+\beta_{10} D_{t} \Delta x_{t} \\
& +\delta_{11} D_{t} x_{t-1}+\delta_{12} D_{t} x_{t-2}+\epsilon_{t}
\end{aligned}
$$

A significância das variáveis dummies $D_{t}$ na equação $(24)$ e seus sinais permitirão concluir se a abertura comercial e os efeitos de curto e longo prazos da produção têm impacto positivo na produtividade.

Estimando (24) por mínimos quadrados ordinários obtém-se suas estimativas e as usuais estatísticas descritas na tabela A.5, no apêndice. Como pode-se perceber

\footnotetext{
${ }^{6}$ Seria possível empregar o teste de Chow com este mesmo objetivo. No entanto, este teste apenas detecta se há ou não quebra estrutural, mas sem especificar se esta quebra se dá devido à mudança no intercepto ou nos coeficientes angulares, ao contrário do que ocorre quando se usa variáveis dummies.
} 
com a ajuda desta tabela, a regressão apresentou um elevado grau de ajustamento, o que pode ser evidenciado através dos indicadores apresentados ( $R^{2}$ ajustado e teste $F$ bastante elevados, Durbin-Watson próximo de 2). As variáveis dummies do intercepto e dos termos de correção de erros $\left(y_{t-1}-x_{t-1}\right)$ e $\left(y_{t-2}-x_{t-2}\right)$, os efeitos de longo prazo, se mostraram significativas de acordo com os testes $t$ no nível de 5\%. Assim, aceita-se a hipótese de que há uma quebra estrutural, em abril de 1990, não só do intercepto, mas também das variáveis que captam os efeitos de longo prazo. Assim, para o período anterior a abril de 1990, obtém-se a seguinte equação estimada:

$$
\begin{aligned}
\Delta y_{t} & =0,40-0,04\left(y_{t-1}-x_{t-1}\right)-0,09\left(y_{t-2}-x_{t-2}\right) \\
& +0,90 \Delta x_{t}-0,03 x_{t-1}-0,06 x_{t-2}
\end{aligned}
$$

Para o período posterior a abril de 1990, dado que as estimativas de $\left(y_{t-1}-\right.$ $\left.x_{t-1}\right)$ e $\left(y_{t-2}-x_{t-2}\right)$ foram praticamente iguais em valores absolutos, ${ }^{7}$ tem-se que a equação estimada foi:

$$
\begin{aligned}
\Delta y_{t} & =0,05+0,45\left[\left(y_{t-1}-x_{t-1}\right)-\left(y_{t-2}-x_{t-2}\right)\right] \\
& +0,88 \Delta x_{t}+0,008 x_{t-1}-0,02 x_{t-2}
\end{aligned}
$$

Observa-se, portanto, para este último período, que quando os desvios de equilíbrio de longo prazo entre a produtividade e produção no período passado excedem esta mesma diferença no período imediatamente anterior, ocorre um aumento da produtividade no presente. Nota-se, também, através da magnitude e significância do coeficiente estimado $(0,45)$ de $\left[\left(y_{t-1}-x_{t-1}\right)-\left(y_{t-2}-x_{t-2}\right)\right]$ na equação (26, que este impacto é razoavelmente forte. Evidentemente, ocorrerá uma queda de produtividade se a diferença entre produtividade e produção no período passado for menor do que esta diferença no período anterior. Esta é justamente uma aproximação da elasticidade produtividade-produção de longo prazo. No período anterior a abril de 1990, a produtividade não é afetada pelos efeitos de longo prazo da produção, já que os coeficientes estimados das variáveis $\left(y_{t-1}-x_{t-1}\right)$ e $\left(y_{t-2}-x_{t-2}\right)$ são não significantes.

Em relação aos impactos de curto prazo, nota-se que o aumento da produção no presente provoca de imediato um aumento de produtividade quase que na mesma

\footnotetext{
${ }^{7}$ Realizou-se um teste de Wald para verificar se os coeficientes de $\left(y_{t-1}-x_{t-1}\right)$ e $\left(y_{t-2}-x_{t-2}\right)$ do modelo, após abril de 1990, são simétricos, ou seja, se $\eta_{1}=-\eta_{2}$. Dado que a estatística do teste obtida foi $F_{0,05}(2,87)=0,06$, concluiu-se pela aceitação de que $\eta_{1}=-\eta_{2}$.
} 
proporção (na verdade 0,9 , que é o coeficiente de $\Delta x_{t}$ na equação $(25)$ ), mas isto só ocorrendo no período anterior a abril de 1990.

Por último, a significância e o sinal positivo estimado do coeficiente da variável dummy $D_{t}$ na equação (24), de acordo com a tabela A.5 no apêndice, permite concluir que os efeitos da abertura da economia brasileira que se deu após o início dos anos 1990 contribuíram de forma positiva para o aumento da produtividade na indústria de transformação.

De posse desses resultados, pode-se inferir que a indústria de transformação do Brasil apresentou, no período 1985-97, um razoável grau de dinamismo, principalmente após a abertura econômica, no início dos anos 1990. Esses resultados podem ser corroborados quando se observa que, no período de janeiro de 1985 até abril de 1990, a produtividade da indústria de transformação do Brasil decresceu a uma taxa média de $0,23 \%$ a.m. (ou -2,77\% a.a.), enquanto que no período que vai de maio de 1990 a dezembro de 1997, a produtividade passou a crescer a uma taxa média de 0,23\% a.m. (ou 2,83\% a.a.), conforme cálculos já apresentados na seção 3 .

Assim, percebe-se que, antes da abertura econômica, somente os efeitos de curto prazo fizeram com que a produtividade decrescesse de forma menos intensa que a produção. E, após a abertura, tanto os efeitos de curto quanto os de longo prazo fizeram com que a produtividade aumentasse em um ritmo bem mais acelerado que a produção industrial. Isto mostra que, à medida que o processo de abertura foi-se consolidando, a indústria de transformação do Brasil deve ter incorporado, de forma mais dinâmica, um conjunto de fatores cujos efeitos implicaram em maiores ganhos de produtividade. Dada a maior necessidade de se produzir produtos mais baratos e de melhor qualidade, a indústria brasileira passou por um processo de reestruturação produtiva, implementado por um significativo grupo de empresas. Esse processo provavelmente permitiu o surgimento de economias de escala, tanto no sentido microeconômico quanto no macroeconômico, que beneficiaram tais empresas, tornando-as mais competitivas. Finalmente, destaca-se que, nos últimos anos, cresceu bastante a importação de máquinas e equipamentos, fator este que pode ter provocado efeitos importantes para a estrutura industrial do país na medida em que há a difusão de novas tecnologias e processos de produção.

\section{Conclusão}

O processo de mudança estrutural da indústria brasileira é, sem dúvida, um processo longo e demorado, mas vem-se consolidando ao longo do tempo, apesar de choques de curto prazo relativamente freqüentes por que tem passado a economia 
brasileira ao longo desta década. Entretanto, as evidências empíricas da lei de Kaldor-Verdoorn, verificadas neste artigo, mostram que a estrutura industrial do Brasil vem apresentando um grau de dinamismo razoável.

Quando se considera todo o período amostral (janeiro de 1985 a dezembro de 1997), observa-se que, através da relação cointegrante entre produtividade-produção - expressão (21) —, o efeito de longo prazo do aumento da produção tem impacto positivo sobre a produtividade da indústria de transformação brasileira, corroborando, assim, a evidência empírica da lei de Kaldor-Verdoorn.

No entanto, como se observa uma quebra estrutural nos primeiros meses dos anos 1990, faz-se importante analisar o período que compreende janeiro de 1985 a abril de 1990, quando a economia brasileira ainda era bastante fechada em relação ao resto do mundo e passava por um processo recessivo e queda de investimentos privados. Neste período, a relação positiva entre produtividade e produção só foi devido aos efeitos de curto prazo, através da demissão de pessoal ocupado e diminuição das horas trabalhadas.

Quanto aos efeitos de longo prazo, pela própria estrutura da economia brasileira vigente naquela época, praticamente não apresentaram nenhuma influência sobre a produtividade. Com efeito, políticas tais como restrição ao comércio via imposição de tarifas, quotas de importação e principalmente, a substituição das importações com base na lei de similar nacional, que objetivava garantir mercado para as empresas nacionais, fizeram com que a indústria nacional não tivesse incentivos para incorporar progressos tecnológicos e ampliar o parque industrial. Estes seriam os efeitos de longo prazo. De fato, observa-se que, na equação (25), os termos de correções de erros que representam os desvios do equilíbrio no longo prazo não são significativos.

Este último resultado serve também para mostrar que a lei de Kaldor-Verdoorn não é simétrica, ou seja, ela só é válida para períodos em que a economia se encontra em fase de expansão, pois, se assim fosse, quando a produção estivesse caindo a produtividade deveria cair mais que proporcionalmente. $\mathrm{O}$ que a evidência tem mostrado é que, em períodos de recessão, no curto prazo, ocorre inicialmente queda do nível de emprego, mas em proporção menor do que a queda de produto. Além do mais, nestes períodos, empresas de baixa produtividade tendem a desaparecer do mercado. Neste sentido, estes fatos podem explicar por que a lei de KaldorVerdoorn não funciona em períodos de queda da produção.

No período seguinte, após o início dos anos 1990, quando ocorre uma maior abertura comercial do país, tanto os efeitos de curto quanto os de longo prazo do aumento da produção são importantes para explicar os ganhos de produtividade da indústria de transformação. De fato, neste período, para enfrentar a 
concorrência dos produtos importados e aumentar a produtividade da indústria nacional, no curto prazo as empresas reduzem o emprego e as horas trabalhadas. No longo prazo, ao perceberem que as mudanças estruturais, tais como abertura e estabilização econômica através do Plano Real, eram definitivas, as empresas se reestruturam, adotando novos processos tecnológicos e inovações organizacionais. Aliado a isso, ocorreram várias fusões de empresas nacionais e acordos com empresas estrangeiras via assistência técnica, prestação de serviços e complementação de linhas de produto.

Por último, os resultados obtidos do modelo econométrico permitem concluir que, após os anos 1990, a produtividade aumentou não só em função dos efeitos de curto e longo prazo do aumento da produção, mas também em função de um maior grau de abertura da economia brasileira.

\section{Referências}

Banerjee, A. (1993). Co-Integration, Error-Correction and the Econometric Analysis of Non-Stationary Data. Oxford University Press, Nova York. et alii.

Bosco, M. A., Castellar, I., Carvalho, J. R. J., \& França, J. M. S. (1997). Padrões de $\beta$-convergência e $\sigma$-convergência: Uma análise da indústria brasileira. Revista Economica do Nordeste, (28):275-288. Numero especial.

Coelli, T., Rao, D. S. P., \& Battese, G. E. (1998). An Introduction to Efficiency and Productivity Analysis. Kluwer Academic Press, Boston, Dorderecht, London.

Färe, R., Grosskopf, S., Norris, M., \& Zhang, Z. (1994). Technical progress, and efficiency change in industrialized countries. American Economic Review, 84(1).

Granger, C. W. J. (1990). The New Palgrave: Econometrics. W. W. Norton, Nova York. Causal inference. In Eatwell, J., Milgate, M. and Newman, P. (orgs.).

Hill, C., Griffiths, W., \& Judge, G. (1999). Econometria. Saraiva, São Paulo.

IBGE (1998). Pesquisa industrial mensal. Capturado em www.ibge.gov.br.

Kaldor, N. (1975). Economic growth and the verdoorn law: A coment of mr. rowthorn's article. The Economic Journal, (85):891-896.

Kenen, P. (1994). The International Economy. Cambridge University Press, Nova York, 3 ed. edition. 
Kennedy, P. (1998). A Guide to Econometrics. MIT Press, Cambridge.

Lima, M. (1993). Produtividade e organização do trabalho: Evolução dos métodos de medição e a influência da organização do processo de trabalho. Master's thesis, UFCE, Fortaleza. Monografia de Bacharelado.

Porter, M. E. (1993). A Vantagem Competitiva Das Nações. Campus, Rio de Janeiro.

Rosa, A. L. e Nogueira, C. A. (1998). Abertura econômica e competitividade da indústria brasileira : Uma análise regional (1985-1997). In Encontro Nacional de Economia, volume 3, pages 1763-1781. Anais.

Rowthorn, R. E. (1975a). A reply to lord kaldor's comment. The Economic Journal, (85):897-901.

Rowthorn, R. E. (1975b). What remains of kaldor's law? The Economic Journal, (85):10-19.

Rowthorn, R. E. (1979). A note on verdoorn's law. The Economic Journal, (89):131-3.

Silva, A. L. \& Laplane, M. F. (1994). Dinâmica recente da indústria brasileira e desenvolvimento competitivo. Economia e Sociedade, (3):81-97.

Verdoorn, P. J. (1951). One empirical law governing the productivity of labor.

Verdoorn, P. J. (1956). Complementarity and long-range projections. Econometrica, (24):429-50.

Verdoorn, P. J. (1980). Verdoorn's law in retrospect: A comment. The Economic Journal, (90):382-5. 


\section{Anexo}

Tabela A.1

Teste de Dickey-Fuller aumentado para LPBRSA

\begin{tabular}{|c|c|c|c|}
\hline Estatística do teste de Dickey-Fuller aumentado & -2.133834 & $\begin{array}{l}\text { Valor crítico a } 1 \%{ }^{*} \\
\text { Valor crítico a } 5 \% \\
\text { Valor crítico a } 10 \%\end{array}$ & $\begin{array}{l}-4,0212 \\
-3,4401 \\
-3,1442\end{array}$ \\
\hline
\end{tabular}

* Valores críticos de MacKinnon para a rejeição da hipótese de raiz unitária.

Equação do Teste de Dickey-Fuller aumentado

MQO // Variável Dependente: D(LPBRSA)

Suposição do teste: Presença de uma Constante e Tendência determinística linear nos dados Amostra (ajustada): 1985:06 1997:12

Observações incluídas: 151 após ajustamentos

\begin{tabular}{|c|c|c|c|c|}
\hline Variável & Coeficiente & Desvio padrão & Estatística $t$ & Probabilidade \\
\hline $\operatorname{LPBRSA}(-1)$ & $-0,088511$ & 0,041480 & $-2,133834$ & 0,0346 \\
\hline $\mathrm{D}(\operatorname{LPBRSA}(-1))$ & 0,000656 & 0,082948 & 0,007911 & 0,9937 \\
\hline $\mathrm{D}(\operatorname{LPBRSA}(-2))$ & $-0,037013$ & 0,083094 & $-0,445437$ & 0,6567 \\
\hline D(LPBRSA $(-3))$ & 0,038899 & 0,082427 & 0,471918 & 0,6377 \\
\hline $\mathrm{D}(\operatorname{LPBRSA}(-4))$ & $-0,178265$ & 0,082088 & $-2,171.649$ & 0,0315 \\
\hline $\mathrm{C}$ & 0,402657 & 0,191230 & 2,105620 & 0,0370 \\
\hline Tendência & $-0,000154$ & 0,000143 & $-1,076155$ & 0,2837 \\
\hline$R^{2}$ & 0,084866 & \multicolumn{2}{|c|}{ Média da var. dependente } & $-0,001236$ \\
\hline$R^{2}$ ajustado & 0,046735 & \multicolumn{2}{|c|}{ D.P. da var. dependente } & 0,060657 \\
\hline E.P. da regressão & 0,059223 & \multicolumn{2}{|c|}{ Critério de Akaike } & $-5,607647$ \\
\hline Soma (resid.) $)^{2}$ & 0,505058 & \multicolumn{2}{|c|}{ Critério de Schwarz } & $-5,467773$ \\
\hline Log máx. verossimilhança & 216,1176 & \multicolumn{2}{|l|}{ Estatística F } & 2,225655 \\
\hline Durbin-Watson & 1,990332 & \multicolumn{2}{|c|}{ Probabilidade (Estatística F) } & 0,043905 \\
\hline
\end{tabular}

Fonte: Estimativas realizadas pelos autores.

Obs.: O número de defasagens do teste de Dickey-Fuller aumentado foi estabelecido automaticamente pelo pacote Econometric Views 2.0. 
Tabela A.2

Teste de Dickey-Fuller aumentado para LPMBRSA

\begin{tabular}{lccc}
\hline Estatística do Teste de Dickey-Fuller aumentado & $-1,679136$ & Valor Crítico a $1 *$ & $-4,0212$ \\
& Valor Crítico a $5 \%$ & $-3,4401$ \\
& Valor Crítico a 10\% & $-3,1442$ \\
\hline
\end{tabular}

*Valores críticos de MacKinnon para a rejeição da hipótese de raiz unitária.

Equação do Teste de Dickey-Fuller Aumentado

MQO // Variável Dependente: D(LPMBRSA)

Suposição do teste: Presença de uma Constante e Tendência determinística linear nos dados

Amostra (ajustada): 1985:06 1997:12

Observações incluídas: 151 após ajustamentos

\begin{tabular}{|c|c|c|c|c|}
\hline Variável & Coeficiente & Desvio padrão & Estatística t & Probabilidade \\
\hline LPMBRSA(-1) & $-0,056669$ & 0,033749 & $-1,679136$ & 0,0953 \\
\hline $\mathrm{D}(\operatorname{LPMBRSA}(-1))$ & $-0,001922$ & 0,082906 & $-0,023184$ & 0,9815 \\
\hline D(LPMBRSA(-2)) & $-0,118140$ & 0,082857 & $-1,425829$ & 0,1561 \\
\hline D(LPMBRSA $(-3))$ & $-0,004784$ & 0,082085 & $-0,058284$ & 0,9536 \\
\hline $\mathrm{D}(\operatorname{LPMBRSA}(-4))$ & $-0,179653$ & 0,081899 & $-2,193596$ & 0,0299 \\
\hline $\mathrm{C}$ & 0,238971 & 0,148365 & 1,610699 & 0,1094 \\
\hline Tendência & 0,000253 & 0,000119 & 2,131716 & 0,0347 \\
\hline$R^{2}$ & 0,083584 & \multicolumn{2}{|c|}{ Média da variável dependente } & 0,002026 \\
\hline$R^{2}$ ajustado & 0,045400 & \multicolumn{2}{|c|}{ D.P. da variável dependente } & 0,056051 \\
\hline E.P. da regressão & 0,054764 & \multicolumn{2}{|c|}{ Critério de Akaike } & $-5,764207$ \\
\hline Soma $(\text { resid })^{2}$ & 0,431865 & \multicolumn{2}{|c|}{ Critério de Schwarz } & $-5,624333$ \\
\hline Log máx. verossimilhança & 227,9379 & \multicolumn{2}{|l|}{ Estatística $F$} & 2,188979 \\
\hline Durbin-Watson & 1,996538 & \multicolumn{2}{|c|}{ Probabilidade (estatística $F$ ) } & 0,047329 \\
\hline
\end{tabular}

Fonte: Estimativas realizadas pelos autores.

Obs.: O número de defasagens do teste de Dickey-Fuller aumentado foi estabelecido automaticamente pelo pacote Econometric Views 2.0. 
Tabela A.3

Teste de cointegração de Johansen para LPMBRSA e LPBRSA

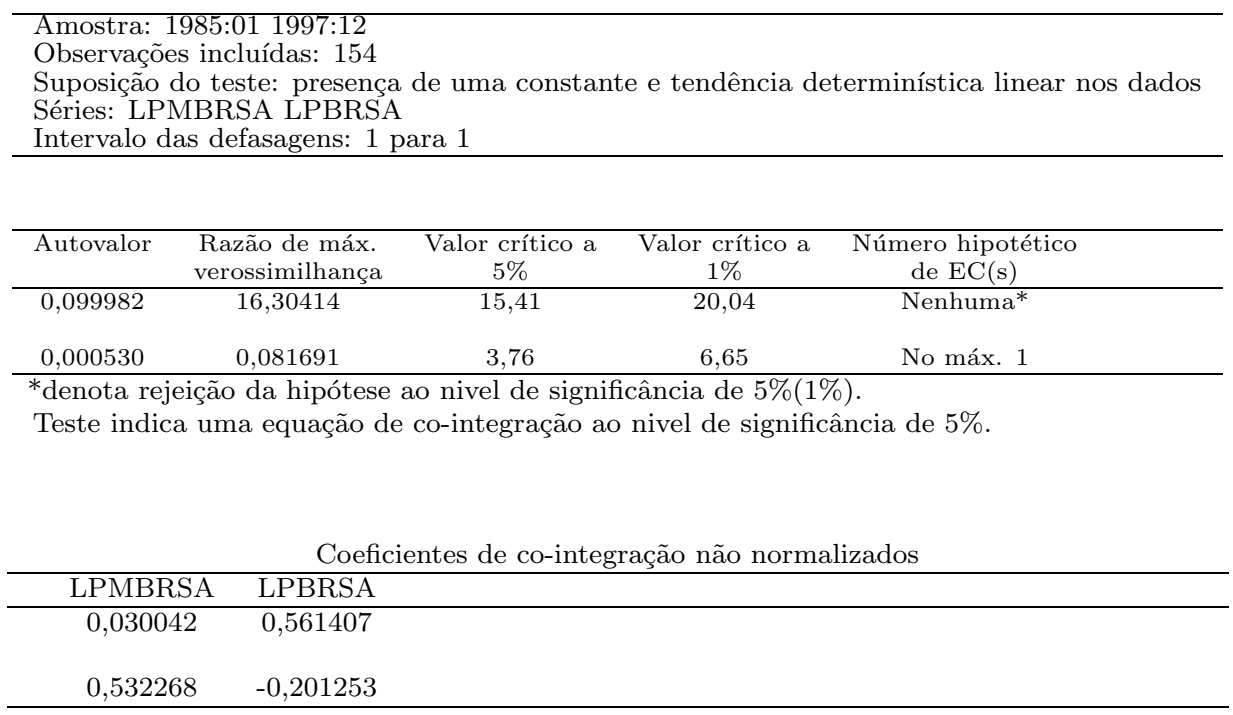

Coeficientes de cointegração normalizados: uma equação de cointegração

\begin{tabular}{|c|c|c|}
\hline LPMBRSA & LPBRSA & $\mathrm{C}$ \\
\hline 1,000000 & $\begin{array}{l}18,68748 \\
(81,6690)\end{array}$ & $-87,27810$ \\
\hline $\begin{array}{l}\text { Log máx. } \\
\text { verossimilhança }\end{array}$ & 861,3212 & \\
\hline
\end{tabular}

Fonte: Estimativas realizadas pelos autores.

Obs.: O número de defasagens do teste de Dickey-Fuller aumentado foi estabelecido automaticamente pelo pacote Econometric Views 2.0.

Tabela A.4

Teste de causalidade de Granger

\begin{tabular}{cccc}
\hline Hipótese Nula (H0) & Teste $F$ & Probabilidade & Rejeição de $H_{0}$ \\
\hline LPBRSA não causa LPMBRSA & 7,90696 & 0,00054 & Sim \\
& & & Não \\
\hline LPMBRSA não causa LPBRSA & $1,07410^{*}$ & 0,34423 & \\
\hline
\end{tabular}

* Não significantes a 5 ou a $10 \%$. 
Tabela A.5

Resultados da regressão

\begin{tabular}{|c|c|c|c|c|}
\hline \multicolumn{5}{|c|}{$\begin{array}{l}\text { MQO // Variável Dependente: D(LPMBRSA) } \\
\text { Amostra (ajustada): 1985:03 1997:12 } \\
\text { Observações incluídas: 154 após ajustamentos. }\end{array}$} \\
\hline Variável & Coeficiente & Desvio padrão & Estatística t & Prob. \\
\hline $\mathrm{C}$ & 0,3954 & 0,07181 & 5,5067 & 0,0000 \\
\hline DI & $-0,0399$ & 0,09694 & $-3,5928$ & 0,0004 \\
\hline (LPMBRSA(-1)-LPBRSA(-1)) & $-0,0868$ & 0,01935 & $-0,3442$ & 0,0000 \\
\hline (LPMBRSA(-2)-LPBRSA(-2)) & $-0,0868$ & 0,02834 & $-0,8069$ & 0,4239 \\
\hline DI*(LPMBRSA(-1)-LPBRSA $(-1))$ & 0,4957 & 0,11603 & 3,1786 & 0,7312 \\
\hline DI*(LPMBRSA(-2)-LPBRSA(-2)) & $-0,3645$ & 0,10763 & $-2,4203$ & 0,4212 \\
\hline D(LPBRSA) & 0,9028 & 0,15595 & 46,656 & 0,0018 \\
\hline DI*D(LPBRSA) & $-0,0227$ & 0,15059 & $-0,8019$ & 0,0168 \\
\hline $\operatorname{LPBRSA}(-1)$ & $-0,0307$ & 0,02304 & $-1,3335$ & 0,1845 \\
\hline $\operatorname{LPBRSA}(-2)$ & $-0,0583$ & 0,02498 & $-2,3359$ & 0,0209 \\
\hline $\mathrm{DI}^{*}(\operatorname{LPBRSA}(-1))$ & 0,0391 & 0,03347 & 1,1680 & 0,2447 \\
\hline DI*(LPBRSA(-2)) & 0,0396 & 0,03217 & 1,2321 & 0,2199 \\
\hline$R^{2}$ & 0,9727 & Média da variá & el dependente & 0,00214 \\
\hline$R^{2}$ ajustado & 0,9706 & D.P. da variáve & dependente & 0,05591 \\
\hline E.P. da regressão & 0,0095 & Critério de Ake & & $-9,22056$ \\
\hline Soma $\left(\right.$ resid.) ${ }^{2}$ & 0,0130 & Critério de Sch & $\operatorname{arz}$ & $-8,98392$ \\
\hline Log máxima verossim. & 503,46 & Estatística $\mathrm{F}$ & & 460,480 \\
\hline Durbin-Watson & 2,0477 & Probabilidade & statística F) & 0,0000 \\
\hline
\end{tabular}

Fonte: Estimativas realizadas pelos autores.

Obs.: O número de defasagens do teste de Dickey-Fuller aumentado foi estabelecido automaticamente pelo pacote Econometric Views 2.0. 